\title{
Serum N-Terminal Pro-B-Type Natriuretic Peptide Levels Are Associated With Functional Capacity in Patients With Peripheral Arterial Disease
}

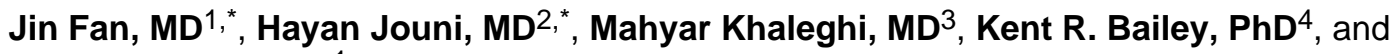 \\ Iftikhar J. Kullo, MD ${ }^{1}$ \\ ${ }^{1}$ Division of Cardiovascular Diseases, Mayo Clinic, Rochester, MN, USA \\ ${ }^{2}$ Department of Medicine, Mayo Clinic, Rochester, MN, USA \\ ${ }^{3}$ Department of Medicine, Georgetown University Hospital/ Washington Hospital Center, \\ Washington, DC, USA \\ ${ }^{4}$ Department of Biomedical Informatics and Statistics, Mayo Clinic, Rochester, MN, USA
}

\section{Abstract}

We hypothesized that higher serum levels of $N$-terminal pro-B-type natriuretic peptide (NT-proBNP) are associated with lower functional capacity in patients with peripheral arterial disease ([PAD] $n=481$, mean age 67,68\% men). Functional capacity was quantified as distance walked on a treadmill for 5 minutes. Patients were divided into 3 groups according to the distance walked: $>144$ yards (group I, $\mathrm{n}=254$ ); 60 to 144 yards (group 2, $\mathrm{n}=80$ ); $<60$ yards or did not walk (group $3, \mathrm{n}=147$ ). The association between NT-pro-BNP levels and the ordinal 3-level walking distance was assessed using multivariable ordinal logistic regression analyses that adjusted for several possible confounding variables. Higher levels of NT-pro-BNP were associated with a lower ordinal walking category independent of possible confounders (odds ratio [OR] 1.51,95\% confidence interval [CI] 1.28-1.77; $P<.001$ ). In conclusion, higher levels of NT-pro-BNP are independently associated with lower functional capacity in patients with PAD and may be a marker of hemodynamic stress in these patients.

\section{Keywords \\ $N$-terminal pro-B-type natriuretic peptide; natriuretic peptides; functional capacity; peripheral arterial disease}

\section{Introduction}

Peripheral arterial disease (PAD) is a relatively common manifestation of systemic atherosclerosis characterized by endothelial dysfunction, arterial stiffness, and stenoses or occlusions of the lower limb arteries. ${ }^{1,2}$ Peripheral arterial disease affects a significant

\footnotetext{
(c) The Author(s) 2011

Corresponding Author: Iftikhar J. Kullo, M.D., Division of Cardiovascular Diseases, Mayo Clinic, 200 First Street SW, Rochester, MN 55905, USA, kullo.iftikhar@mayo.edu.

*The authors JF and HJ contributed equally to this work.

This work was previously presented as an abstract at The American College of Cardiology 59th Scientific Session. Atlanta, GA; March 14-16, 2010.
}

Declaration of Conflicting Interests: The author(s) declared no potential conflicts of interest with respect to the research, authorship, and/or publication of this article. 
proportion of elderly populations worldwide ${ }^{3,4}$ and is associated with adverse cardiovascular events including myocardial infarction, stroke, and death. ${ }^{5}$ As the proportion of elderly people in the population increases, PAD will become an even greater public health burden unless steps are taken to reduce the prevalence of modifiable risk factors such as smoking. Functional disability is common among patients with PAD and leads to impaired quality of life. ${ }^{6,7}$ One of the major goals of PAD management is to improve functional capacity. A better understanding of the mechanisms underlying impaired functional capacity is needed to develop effective interventions and improve the quality of life in patients with PAD.

In previous studies, ${ }^{8,9}$ we demonstrated that arterial stiffness is associated with reduced functional capacity in patients with PAD. $N$-terminal pro-B-type natriuretic peptide (NTpro-BNP), a marker of left ventricular and hemodynamic stress, plays important roles in modifying cardiac load and reparative vascular remodeling. ${ }^{10}$ Several studies have shown NT-pro-BNP to be elevated in patients with $\mathrm{PAD}^{11-13}$ and to be associated with the severity of PAD based on Fontaine classification. ${ }^{14}$ Moreover, levels of NT-pro-BNP are predictive of 5-year mortality in patients with PAD. ${ }^{15}$ The relationship between NT-pro-BNP and functional capacity has not been studied in patients with PAD. Since arterial stiffness leads to increased hemodynamic and cardiac burden, we hypothesized that higher levels of NTpro-BNP would be associated with lower functional capacity in patients with PAD. We tested this hypothesis by measuring serum NT-pro-BNP levels in patients referred for outpatient noninvasive lower extremity arterial evaluation.

\section{Methods}

\section{Study Population}

The study protocol was approved by the Institutional Review Board of the Mayo Clinic and all patients gave informed written consent. Between December 2006 and December 2008, 548 patients with PAD referred to the Mayo Clinic outpatient noninvasive vascular laboratory were recruited to participate in a study of genetic and proteomic biomarkers of PAD. Peripheral arterial disease was defined based on having a resting or post-exercise ankle-brachial index (ABI) $\$ 0.9$, presence of poorly compressible arteries (ABI >1.4; or ankle blood pressure [BP] $>255 \mathrm{~mm} \mathrm{Hg}$ ), or prior history of lower extremity revascularization. We identified patients with heart failure by screening the electronic medical record (EMR) for the corresponding International Classification of Diseases, Ninth Revision, Clinical Modification (ICD-9-CM) code (428.*) within the time frame of 1 year centered upon the date of sample collection. The medical record of these patients was reviewed and they were excluded if they had an ejection fraction $<50 \%$, diastolic dysfunction grades 3 and 4, moderate/severe valvular abnormalities, presence of Framingham criteria for heart failure, or other structural heart diseases. We eventually excluded 32 patients with heart failure based on the above algorithm in addition to patients who had nonatherosclerotic vascular diseases $(n=35)$.

\section{Noninvasive Lower Extremity Arterial Evaluation}

Resting and post-exercise ABI were measured by vascular technicians in the noninvasive vascular laboratory using an established protocol. ${ }^{16}$ The higher of the 2 brachial systolic BPs was used to calculate the $\mathrm{ABI}$ at the posterior tibial artery and the dorsalis pedis artery in each leg. For each patient, the lowest resting $\mathrm{ABI}$ and the $\mathrm{ABI}$ at 1 minute after exercise were used in the analyses. Functional capacity was assessed using a fixed-grade motorized treadmill (Treadmill Proform, Icon Health \& Fitness, Logan, Illinois). A 5-minute treadmill test with a speed of 1 to $2 \mathrm{mph}$ and a fixed grade of $10^{\circ}$ with continuous electrocardiogram monitoring was performed to measure post-exercise ABI as well as walking distance.

Functional capacity was estimated in terms of distance covered in 5 minutes for those who 
walked less than 5 minutes. ${ }^{17,18}$ For patients who walked the full 5 minutes, the walking distance is "right censored" and the censoring distance is different depending on the walking speed. Therefore, the functional capacity for these patients was imputed as described in the statistical analysis section. Per laboratory protocol, patients with severe PAD $(\mathrm{ABI}<0.5)$ or poorly compressible arteries did not undergo the treadmill test.

\section{Cardiovascular Risk Factors and Comorbidities}

The EMR was reviewed to ascertain age, sex, body mass index (BMI), systolic and diastolic BP, cardiovascular risk factors (hypertension, diabetes, dyslipidemia, and smoking status), comorbidities (coronary heart disease, cerebrovascular disease, or heart failure), and statin use. The laboratory values closest to the date of sample collection were also extracted from the EMR including serum creatinine, fasting, glucose, triglycerides, and total and highdensity lipoprotein (HDL) cholesterol. Smoking status was classified as "ever" or "never." The diagnosis of hypertension was established either with $2 \mathrm{BP}$ readings of $\geq 140 / 90 \mathrm{~mm} \mathrm{Hg}$ within 3 months or a prior diagnosis of hypertension and current treatment with antihypertensive medications. Similarly, diabetes was diagnosed if the patient had a fasting glucose measurement $>126 \mathrm{mg} / \mathrm{dL}$ or a random glucose $>200 \mathrm{mg} / \mathrm{dL}$ or had a prior diagnosis of diabetes, and was on treatment with oral hypoglycemic agents or insulin. Dyslipidemia was defined as total cholesterol $>200 \mathrm{mg} / \mathrm{dL}$ or HDL cholesterol $<40 \mathrm{mg} / \mathrm{dL}$ (men), $<45 \mathrm{mg}$ / $\mathrm{dL}$ (women) or triglycerides $>150 \mathrm{mg} / \mathrm{dL}$, or use of a lipid-lowering medication. Estimated glomerular filtration rate (eGFR) was calculated using the abbreviated equation from the "Modification of Diet in Renal Disease study" by Levey et al. ${ }^{19}$

\section{N-Terminal Pro-B-Type Natriuretic Peptide Assay}

Samples of venous blood were taken after 20 to 30 minutes of rest in an upright sitting position. Serum samples were immediately frozen and stored at $-80^{\circ} \mathrm{C}$ until assessment. Serum NT-pro-BNP was measured by the commercially available Roche electrochemiluminescence immunoassay. Intra-assay and interassay precision was assessed using BioRad Cardiac controls. The intra-assay coefficient of variations (CVs) were $1.1 \%$ and $1.3 \%$ at 122 and $3629 \mathrm{pg} / \mathrm{mL}$, respectively, and the interassay CVs were $3.1 \%$ and $1.8 \%$ at 121 and $3635 \mathrm{pg} / \mathrm{mL}$, respectively.

\section{Statistical Analysis}

Continuous data were summarized as mean \pm standard deviation (SD) or median (interquartile ranges; IQR) and categorical data were expressed as percentages (\%). Serum levels of NT-pro-BNP were log transformed because of skewed distribution. For patients who completed the 5-minute walk $(\mathrm{n}=254)$, we imputed mean walking times since discarding them or simply treating them as the actual walking distance could lead to biased results. Imputation was based on normal theory calculations using speed-specific truncated mean, variance, and estimated normal truncation point (a detailed description of the imputation procedure is provided in Appendix A). The imputed walking times for patients who completed the 5-minute treadmill test at walking speeds of $1,1.5$, and $2.0 \mathrm{mph}$ were $6.24,6.60$, and 7.18 minutes, respectively. This was consistent with an increasing proportion of patients finishing the 5-minute walk at speeds of 1.0, 1.5, and 2.0, respectively. Walking distances were then calculated as imputed walking time multiplied by walking speed.

The association of NT-pro-BNP levels with functional capacity was assessed by ordinal logistic as well as linear regression analyses. Regression analyses were performed in a hierarchical fashion, sequentially adjusting for potential confounding variables. Patients were divided into 3 ordinal categories according to their functional capacity (walked $<60$ yards or did not walk, walked 60-144 yards, or walked >144 yards), and predictors of functional capacity were analyzed by ordinal logistic regression analysis. In the subgroup of 
patients who walked $(\mathrm{n}=352)$, multivariable linear regression analysis was performed using walking distance (observed or imputed) as the dependent variable. The variables adjusted for included age, sex, BMI, smoking status, diabetes, hypertension, dyslipidemia, coronary heart disease/cerebrovascular disease, eGFR, statin use, pulse pressure, and resting ABI. A 2sided $P<.05$ was deemed significant. Statistical analyses were carried out using SAS v 9.1.3 (SAS Institute, Cary NC) software package.

\section{Results}

The clinical characteristics of the 481 study participants by strata of functional capacity are summarized in Table 1; $31 \%$ were diabetic, $72 \%$ were hypertensive, $85 \%$ had dyslipidemia, $54 \%$ had history of coronary heart disease, and $34 \%$ had history of cerebrovascular disease. Patients in the lower functional capacity categories had higher levels of NT-pro-BNP, were older, had higher prevalence of diabetes, and lower prevalence of history of smoking. Overall, $52 \%(\mathrm{n}=254$ patients $)$ walked $>144$ yards; $17 \%(\mathrm{n}=80)$ walked 60 to 144 yards; and $31 \%(\mathrm{n}=147)$ walked $<60$ yards or did not walk. In the subgroup of patients who walked ( $\mathrm{n}=352$ ), the mean walking distance (observed or otherwise censored) was $202 \pm 75$ yards and the mean walking time was $4.3 \pm 1$ minutes. Among 129 patients who did not walk, 46\% $(n=60)$ had severe PAD and 54\% $(n=69)$ had poorly compressible arteries.

The median (IQR) levels of NT-pro-BNP were significantly higher among those with poorer functional capacity (109 [52-297], 177 [71-411], and 425 [116-1,214] pg/mL; $P<.001$, in the 3 groups respectively, Table 1). As shown in Figure 1, levels of NT-pro-BNP were associated with functional capacity and were significantly higher in groups 2 and 3 compared with group 1 . Only $20 \%$ of patient in the first quartile of NT-pro-BNP levels $(<64$ $\mathrm{pg} / \mathrm{mL}$ ) had poor functional capacity (group 3 ), whereas approximately $60 \%$ of those who were in the fourth quartile of NT-pro-BNP levels $(>520 \mathrm{pg} / \mathrm{mL})$ had poor functional capacity. In multivariable ordinal logistic regression analysis, a 1 unit increase in log NTpro-BNP levels was associated with an OR of 1.53 for having lower functional capacity (95\% CI: 1.33-1.76; $P<.001$ ) after adjustment for age, sex, and BMI. This association remained significant $(P<.001)$ in all of the subsequent models that also adjusted for smoking status, diabetes, hypertension, coronary heart disease/cerebrovascular disease, eGFR, statin use, pulse pressure, and resting ABI. Table 2 demonstrates the sequential adjustment in each of the statistical models with the respective $P$ values. In addition, older age $(P=.008)$ was also a significant predictor of lower functional capacity.

In multivariable linear regression analyses limited to those patients who walked, lower walking distance was significantly associated with higher levels of NT-pro-BNP $(\beta \pm$ standard error $[\mathrm{SE}]:-19.0 \pm 5.2 ; P<.001)$. When adjusted for age, sex, and BMI, the association was attenuated but remained significant $(\beta+\mathrm{SE}:-11.9 \pm 5.4 ; P=.038)$. In a model incorporating additional possible confounding variables, NT-pro-BNP remained significantly associated with lower functional capacity $(\beta \pm \mathrm{SE} ;-11.6+5.9 ; P=.049$; Table $3)$. Other predictors of lower functional capacity in the final model were older age $(P<$. $001)$, female sex $(P<.001)$, greater BMI $(P=.002)$, and lower resting ABI $(P=.002)$.

\section{Discussion}

The main finding of this study is that higher levels of NT-pro-BNP are associated with lower functional capacity in patients with PAD independent of potential confounders including age, sex, BMI, smoking status, diabetes, hypertension, coronary heart disease, cerebrovascular disease, dyslipidemia, statin use, renal function, pulse pressure, and resting ABI. To the best of our knowledge, the present study is the first to show an association between serum levels of NT-pro-BNP and functional capacity of patients with PAD. Higher 
levels of NT-pro-BNP in patients with PAD may reflect impaired cardiac reserve and inadequate peripheral perfusion due to advanced systemic atherosclerosis of the coronary circulation as well as the peripheral arterial bed. Consistent with previous studies, we noted that older age, female sex, higher BMI, and lower resting ABI were associated with impaired functional capacity in patients with PAD. ${ }^{8,9,20}$

$\mathrm{N}$-terminal pro-B-type natriuretic peptide is a key cardiovascular peptide hormone mainly secreted from ventricular cardiomyocytes in response to increased transmural wall tension due to ventricular stress, hypertrophy, or volume overload. ${ }^{21,22}$ It is widely accepted that patients with PAD have endothelial dysfunction and increased arterial stiffness with hemodynamic consequences of increased flow resistance and systolic workload, and reduced diastolic perfusion of the left ventricle. ${ }^{23-26} \mathrm{We}$ and others have shown that increased arterial stiffness is associated with decreased functional capacity in patients with PAD. ${ }^{8,9}$ In addition, arterial stiffness is known to increase in the presence of risk factors that predispose to coronary atherosclerosis and that may in turn result in subclinical myocardial ischemia, ${ }^{27,28}$ with a subsequent increase in cardiac filling pressures, ventricular workload, and reduction in cardiac reserve. Diminished cardiac reserve combined with lower extremity ischemia due to arterial stiffening, stenosis, or occlusion may limit functional capacity in patients with PAD. ${ }^{20}$ Simultaneously, the increased afterload, ventricular stiffness, and filling pressures will eventually lead to increased ventricular wall stress/tension and NT-proBNP secretion. ${ }^{29,30}$ The aforementioned factors will collectively lead to decreased functional reserve of the left ventricle, reduced exercise tolerance, and diminished walking capacity.

Moreover, BNP has been recently shown to be secreted from the skeletal muscles in mice in response to hind limb ischemia. ${ }^{31}$ Therefore, the increased levels of NT-pro-BNP in patients with PAD could be dual in origin with ventricular stress being the central and probably the main site of BNP production along with the peripheral production of BNP from the affected ischemic skeletal muscles. ${ }^{10,31}$ Figure 2 summarizes the key mechanisms that might link increased NT-pro-BNP levels with decreased functional capacity in patients with PAD.

Natriuretic peptides may have beneficial effects in patients with PAD by promoting angiogenesis, modifying vascular endothelial function, reducing cardiac load, and improving blood supply to the legs by diuretic and vasodilatory effects. ${ }^{31-34}$ In animals, low-dose infusions of atrial natriuretic peptide (ANP) over 48 hours reduced peripheral vascular resistance and BP. ${ }^{35}$ Transgenic mice that overexpress BNP in response to hind limb ischemia have accelerated vascular regeneration following experimental femoral artery ligation. ${ }^{36}$ More recently, Park et al found that infusion of recombinant human ANP in patients with PAD increased $\mathrm{ABI}$ and improved rest pain, pain-free walking distance, and ulcer healing. ${ }^{33}$ Further randomized studies are needed to evaluate the therapeutic potential of natriuretic peptides in patients with PAD.

An alternative approach to improving functional capacity in patients with PAD may be to reduce arterial stiffness which in turn will result in decreased hemodynamic stress. Statins have been shown to decrease arterial stiffness, ${ }^{37}$ improve endothelial function, ${ }^{38}$ and increase functional capacity in patients with PAD. ${ }^{39}$ In a meta-analysis of 43 clinical trials, statins increased walking distance by nearly $160 \mathrm{~m} .{ }^{40}$ Angiotensin-converting enzyme (ACE) inhibitors appear to reduce arterial stiffness by decreasing collagen deposition and increasing fibrillin 1 production in the vascular wall. ${ }^{41}$ Angiotensin-converting enzyme inhibitors have been shown to increase walking distance in patient with $\mathrm{PAD},{ }^{42}$ and $\mathrm{ACE}$ inhibitor-related decreases in arterial stiffness have been proposed as the underlying mechanism for improvement in functional capacity. In 40 patients with PAD (mean age, 66), 
those receiving ramipril for 24 weeks doubled their walking time compared to patients receiving placebo, with concomitant improvement in arterial stiffness parameters. ${ }^{42}$

Although patients with PAD may benefit from increased circulating levels of BNP, such levels represent increased hemodynamic stress and possibly ongoing skeletal muscle ischemia. Levels of NT-pro-BNP are also predictive of 5-year mortality in patients with PAD. ${ }^{15}$ Levels of NT-pro-BNP could be useful in routine clinical visits for personalized therapy to guide further diagnostic and therapeutic interventions including revascularization and regular exercise programs. Further studies are needed to demonstrate whether treatment strategies guided by NT-pro-BNP levels will decrease morbidity and mortality in patients with PAD and whether NT-pro-BNP will find a place in the routine clinical stratification of risk among such patients.

\section{Strengths and Limitations}

Our study has several potential limitations. Patients with PAD were recruited from patients referred to the noninvasive vascular laboratory and some degree of referral bias is likely present. The assessment of heart failure was based on the review of EMR. Therefore, there is a chance that part of observed association is related to undiagnosed heart failure or presence of patients with asymptomatic heart failure. However, the prevalence of heart failure in this study $(6 \%)$ is similar to the previously reported prevalence in patients with PAD. ${ }^{15,43}$ To estimate the functional capacity more objectively, imputed walking distance was used in the analyses of patients who completed the exercise test. Also, patients with severe PAD and poorly compressible arteries did not walk per our laboratory protocol and this may have biased the ordinal logistic regression analysis results. However, in multivariable linear regression analyses that included only patients who walked, higher levels of NT-pro-BNP remained significantly associated with lower walking distance after adjustment for possible confounding variables.

\section{Conclusions}

We demonstrate that higher levels of serum NT-pro-BNP are independently associated with impaired functional capacity in patients with PAD. These findings suggest that elevated levels of NT-pro-BNP may be a marker of hemodynamic stress and atherosclerotic burden in patients with PAD. The underlying pathophysiological mechanisms and clinical implications of these findings warrant further investigation.

\section{Acknowledgments}

The authors thank Guanghui Liu for his help with statistical analyses.

Funding: The author(s) disclosed receipt of the following financial support for the research, authorship, and/or publication of this article: supported by grants HL-81331 and UL1-RR024150 (Center for Translational Science Activities) from the National Institutes of Health, Bethesda, Maryland, and by a Marriott Award for Individualized Medicine.

\section{Appendix A: Walking Distance Imputation Calculations}

Suppose we observe that a proportion $P$ finishes the 5-minute walk in a particular treadmill speed group.

Let $C=\Phi^{-1}(P)$ and let $\Phi(C)=P$ 
and let $\varphi(\mathrm{C})$ designate the normal density at this truncation/censoring point. Then the mean and variance of the observed (truncated) distribution of $X$ can be related to the mean and variance of the unobserved untruncated normal distribution via the following relationships:

$$
\begin{gathered}
E(X)=\mu+\sigma[C(1-\Phi)-\phi] \\
\frac{\operatorname{Var}(X)}{\sigma^{2}}=C^{2} \cdot \Phi(1-\Phi)+\Phi+C \cdot \phi-2 \cdot C \cdot \Phi \cdot \phi-\phi^{2}
\end{gathered}
$$

We then equate the observed mean and variance to the values in the left side of these 2 equations and solve for the underlying mean and variance parameters $\mu$ and $\sigma^{2}$.

$$
\begin{gathered}
\widehat{\sigma}^{2}=\frac{\operatorname{Var}(X)}{\left(C^{2} \cdot \Phi(1-\Phi)+\Phi+C \cdot \phi-2 \cdot C \cdot \Phi \cdot \phi-\phi^{2}\right)} \\
\widehat{\mu}=E(X)-\widehat{\sigma}[C(1-\Phi)-\phi]
\end{gathered}
$$

Finally, we use these estimated underlying normal mean and variance to calculate the expected residual time within each walking speed for all finishers.

$$
E(T \mid(T>5))=\widehat{\mu}+\widehat{\sigma} \cdot \frac{\phi}{(1-\Phi)}
$$

\section{References}

1. Belch JJ, Topol EJ, Agnelli G, et al. Critical issues in peripheral arterial disease detection and management: a call to action. Arch Intern Med. 2003; 163(8):884-892. [PubMed: 12719196]

2. Weitz JI, Byrne J, Clagett GP, et al. Diagnosis and treatment of chronic arterial insufficiency of the lower extremities: a critical review. Circulation. 1996; 94(11):3026-3049. [PubMed: 8941154]

3. Criqui MH, Denenberg JO, Langer RD, Fronek A. The epidemiology of peripheral arterial disease: importance of identifying the population at risk. Vasc Med. 1997; 2(3):221-226. [PubMed: 9546971]

4. Murabito JM, D'Agostino RB, Silbershatz H, Wilson WF. Intermittent claudication. A risk profile from the framingham heart study. Circulation. 1997; 96(1):44-49. [PubMed: 9236415]

5. Gornik HL, Creager MA. Contemporary management of peripheral arterial disease: I. cardiovascular risk-factor modification. Cleve Clin J Med. 2006; 73(suppl 4):S30-S37. [PubMed: 17385389]

6. McDermott MM, Greenland P, Liu K, et al. Leg symptoms in peripheral arterial disease: associated clinical characteristics and functional impairment. JAMA. 2001; 286(13):1599-1606. [PubMed: 11585483]

7. Newman AB, Naydeck BL, Sutton-Tyrrell K, Polak JF, Kuller LH. The role of comorbidity in the assessment of intermittent claudication in older adults. J Clin Epidemiol. 2001; 54(3):294-300. [PubMed: 11223327]

8. Amoh-Tonto CA, Malik AR, Kondragunta V, Ali Z, Kullo IJ. Brachial-ankle pulse wave velocity is associated with walking distance in patients referred for peripheral arterial disease evaluation. Atherosclerosis. 2009; 206(1):173-178. [PubMed: 19278681]

9. Brewer LC, Chai HS, Bailey KR, Kullo IJ. Measures of arterial stiffness and wave reflection are associated with walking distance in patients with peripheral arterial disease. Atherosclerosis. 2007; 191(2):384-390. [PubMed: 16730015]

10. Tokudome T, Kishimoto I, Yamahara K, et al. Impaired recovery of blood flow after hind-limb ischemia in mice lacking guanylyl cyclase-a, a receptor for atrial and brain natriuretic peptides. Arterioscler Thromb Vasc Biol. 2009; 29(10):1516-1521. [PubMed: 19628785] 
11. Jouni H, Rodeheffer RJ, Kullo IJ. Increased serum N-terminal pro-B-type natriuretic peptide levels in patients with medial arterial calcification and poorly compressible leg arteries. Arterioscler Thromb Vasc Biol. 2011; 31(1):197-202. [PubMed: 20947817]

12. Montagnana M, Lippi G, Fava C, et al. Ischemia-modified albumin and NT-prohormone-brain natriuretic peptide in peripheral arterial disease. Clin Chem Lab Med. 2006; 44(2):207-212. [PubMed: 16475909]

13. Svensson P, de Faire U, Niklasson U, Hansson LO, Ostergren J. Plasma NT-pro-BNP concentration is related to ambulatory pulse pressure in peripheral arterial disease. Blood Press. 2005; 14(2):99-106. [PubMed: 16036487]

14. Dieplinger B, Poelz W, Haltmayer M, Mueller T. Association of adiponectin and amino terminal proBNP in peripheral arterial disease. Clin Chim Acta. 2007; 377(1-2):192-197. [PubMed: 17112494]

15. Mueller T, Dieplinger B, Poelz W, Endler G, Wagner OF, Haltmayer M. Amino-terminal pro-Btype natriuretic peptide as predictor of mortality in patients with symptomatic peripheral arterial disease: 5-year follow-up data from the linz peripheral arterial disease study. Clin Chem. 2009; 55(1):68-77. [PubMed: 18988753]

16. Santos S, Rooke TW, Bailey KR, McConnell JP, Kullo IJ. Relation of markers of inflammation (Creactive protein, white blood cell count, and lipoprotein-associated phospholipase a2) to the anklebrachial index. Vasc Med. 2004; 9(3):171-176. [PubMed: 15675180]

17. Gardner AW, Skinner JS, Cantwell BW, Smith LK. Progressive vs single-stage treadmill tests for evaluation of claudication. Med Sci Sports Exerc. 1991; 23(4):402-408. [PubMed: 2056896]

18. Hirsch AT, Haskal ZJ, Hertzer NR, et al. ACC/AHA 2005 guidelines for the management of patients with peripheral arterial disease (lower extremity, renal, mesenteric, and abdominal aortic): executive summary a collaborative report from the american association for vascular surgery/ society for vascular surgery, society for cardiovascular angiography and interventions, society for vascular medicine and biology, society of interventional radiology, and the ACC/AHA task force on practice guidelines (writing committee to develop guidelines for the management of patients with peripheral arterial disease) endorsed by the american association of cardiovascular and pulmonary rehabilitation; national heart, lung, and blood institute; society for vascular nursing; transatlantic inter-society consensus; and vascular disease foundation. J Am Coll Cardiol. 2006; 47(6):1239-1312. [PubMed: 16545667]

19. Levey AS, Coresh J, Greene T, et al. Using standardized serum creatinine values in the modification of diet in renal disease study equation for estimating glomerular filtration rate. Ann Intern Med. 2006; 145(4):247-254. [PubMed: 16908915]

20. McDermott MM, Greenland P, Liu K, et al. The ankle brachial index is associated with leg function and physical activity: the walking and leg circulation study. Ann Intern Med. 2002; 136(12):873-883. [PubMed: 12069561]

21. Luchner A, Burnett JC Jr, Jougasaki M, Hense HW, Riegger GA, Schunkert H. Augmentation of the cardiac natriuretic peptides by beta-receptor antagonism: evidence from a population-based study. J Am Coll Cardiol. 1998; 32(7):1839-1844. [PubMed: 9857860]

22. Maisel AS, Krishnaswamy P, Nowak RM, et al. Rapid measurement of B-type natriuretic peptide in the emergency diagnosis of heart failure. N Engl J Med. 2002; 347(3):161-167. [PubMed: 12124404]

23. Cheng EM, Asch SM, Brook RH, et al. Suboptimal control of atherosclerotic disease risk factors after cardiac and cerebrovascular procedures. Stroke. 2007; 38(3):929-934. [PubMed: 17255549]

24. Dao HH, Essalihi R, Bouvet C, Moreau P. Evolution and modulation of age-related medial elastocalcinosis: impact on large artery stiffness and isolated systolic hypertension. Cardiovasc Res. 2005; 66(2):307-317. [PubMed: 15820199]

25. Ferreira I, Twisk JW, Van Mechelen W, Kemper HC, Stehouwer CD. Current and adolescent levels of cardiopulmonary fitness are related to large artery properties at age 36: the amsterdam growth and health longitudinal study. Eur J Clin Invest. 2002; 32(10):723-731. [PubMed: 12406019]

26. O'Rourke MF. Mechanical principles. Arterial stiffness and wave reflection. Pathol Biol (Paris). 1999; 47(6):623-633. [PubMed: 10472073] 
27. Her K, Choi C, Park Y, Shin H, Won Y. Concomitant peripheral artery disease and asymptomatic coronary artery disease: a management strategy. Ann Vasc Surg. 2008; 22(5):649-656. [PubMed: 18504099]

28. McDermott MM, Liu K, Criqui MH, et al. Ankle-brachial index and subclinical cardiac and carotid disease: the multi-ethnic study of atherosclerosis. Am J Epidemiol. 2005; 162(1):33-41. [PubMed: 15961584]

29. Lester SJ, Tajik AJ, Nishimura RA, Oh JK, Khandheria BK, Seward JB. Unlocking the mysteries of diastolic function: deciphering the rosetta stone 10 years later. J Am Coll Cardiol. 2008; 51(7): 679-689. [PubMed: 18279730]

30. Rubattu S, Sciarretta S, Valenti V, Stanzione R, Volpe M. Natriuretic peptides: an update on bioactivity, potential therapeutic use, and implication in cardiovascular diseases. Am J Hypertens. 2008; 21(7):733-741. [PubMed: 18464748]

31. Kuhn M, Volker K, Schwarz K, et al. The natriuretic peptide/guanylyl cyclase-a system functions as a stress-responsive regulator of angiogenesis in mice. J Clin Invest. 2009; 119(7):2019-2030. [PubMed: 19487812]

32. Chen H, Levine YC, Golan DE, Michel T, Lin AJ. Atrial natriuretic peptide-initiated cGMP pathways regulate vasodilator-stimulated phosphoprotein phosphorylation and angiogenesis in vascular endothelium. J Biol Chem. 2008; 283(7):4439-4447. [PubMed: 18079117]

33. Park K, Itoh H, Yamahara K, et al. Therapeutic potential of atrial natriuretic peptide administration on peripheral arterial diseases. Endocrinology. 2008; 149(2):483-491. [PubMed: 17991722]

34. Shmilovich H, Ben-Shoshan J, Tal R, et al. B-type natriuretic peptide enhances vasculogenesis by promoting number and functional properties of early endothelial progenitor cells. Tissue Eng Part A. 2009; 15(9):2741-2749. [PubMed: 19275472]

35. Charles CJ, Espiner EA, Richards AM. Cardiovascular actions of ANF: contributions of renal, neurohumoral, and hemodynamic factors in sheep. Am J Physiol. 1993; 264(3 pt 2):R533-R538. [PubMed: 8096125]

36. Yamahara K, Itoh H, Chun TH, et al. Significance and therapeutic potential of the natriuretic peptides/cGMP/cGMP-dependent protein kinase pathway in vascular regeneration. Proc Natl Acad Sci U S A. 2003; 100(6):3404-3409. [PubMed: 12621153]

37. Mukherjee S, Mukhopadhyay P, Pandit K, Chowdhury S. Atorvastatin improves arterial stiffness in normotensive normolipidaemic persons with type 2 diabetes. J Indian Med Assoc. 2008; 106(11):716-719. [PubMed: 19368096]

38. Laufs U, La Fata V, Plutzky J, Liao JK. Upregulation of endothelial nitric oxide synthase by HMG coA reductase inhibitors. Circulation. 1998; 97(12):1129-1135. [PubMed: 9537338]

39. Aronow WS, Nayak D, Woodworth S, Ahn C. Effect of simvastatin versus placebo on treadmill exercise time until the onset of intermittent claudication in older patients with peripheral arterial disease at six months and at one year after treatment. Am J Cardiol. 2003; 92(6):711-712. [PubMed: 12972114]

40. Momsen AH, Jensen MB, Norager CB, Madsen MR, Vestersgaard-Andersen T, Lindholt JS. Drug therapy for improving walking distance in intermittent claudication: a systematic review and metaanalysis of robust randomised controlled studies. Eur J Vasc Endovasc Surg. 2009; 38(4):463-474. [PubMed: 19586783]

41. Ahimastos AA, Natoli AK, Lawler A, Blombery PA, Kingwell BA. Ramipril reduces large-artery stiffness in peripheral arterial disease and promotes elastogenic remodeling in cell culture. Hypertension. 2005; 45(6):1194-1199. [PubMed: 15897362]

42. Ahimastos AA, Dart AM, Lawler A, Blombery PA, Kingwell BA. Reduced arterial stiffness may contribute to angiotensin-converting enzyme inhibitor induced improvements in walking time in peripheral arterial disease patients. J Hypertens. 2008; 26(5):1037-1042. [PubMed: 18398348]

43. Anand RG, Ventura HO, Mehra MR. Is heart failure more prevalent in patients with peripheral arterial disease? A meta-analysis. Congest Heart Fail. 2007; 13(6):319-322. [PubMed: 18046089] 

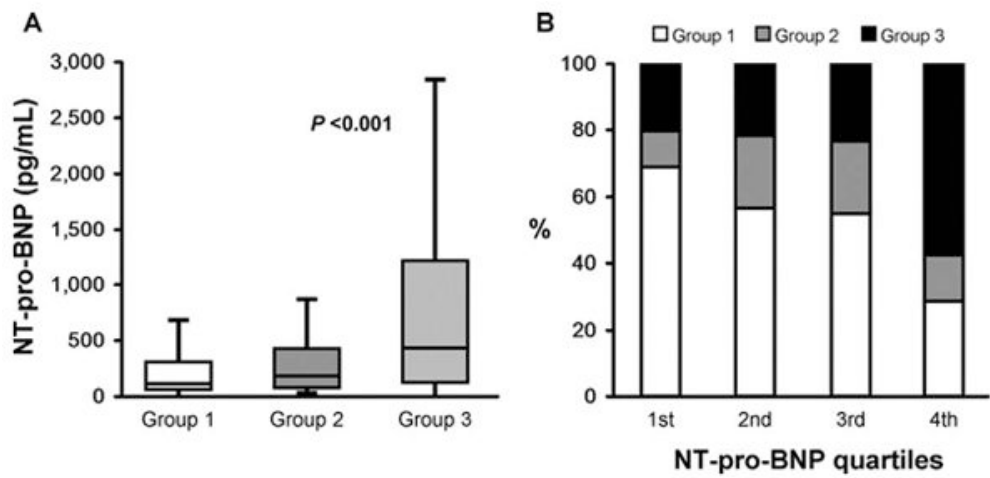

Figure 1.

A, Box and whisker plots comparing serum NT-pro-BNP levels among the study groups. Patients in group 1 walked >144 yards compared with group 2 (60-144 yards) and group 3 ( $<60$ yards or did not walk due to ABI $<0.5$ or poorly compressible arteries). B, The proportion of 3 functional groups in each quartile of NT-pro-BNP levels. NT-pro-BNP indicates $N$-terminal pro-B-type natriuretic peptide; ABI, ankle-brachial index. 


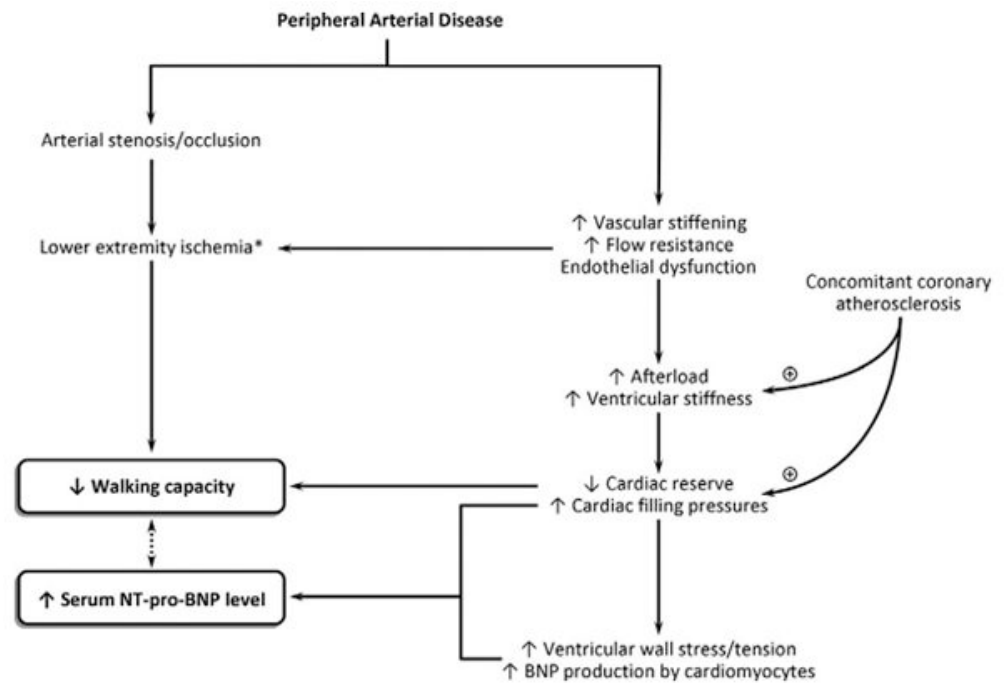

Figure 2.

Possible mechanisms underlying the association between walking capacity and $N$-terminal pro-B-type natriuretic peptide levels in patients with peripheral arterial disease. Asterisk indicates lower extremity ischemia may cause B-type natriuretic peptide secretion from satellite cells within the ischemic skeletal muscles. 
Table 1

Baseline Characteristics of the Study Patients ${ }^{a}$

\begin{tabular}{|c|c|c|c|}
\hline & Group $1^{b}, \mathrm{n}=254$ & Group $2^{c}, \mathrm{n}=80$ & Group $3^{d}, \mathrm{n}=147$ \\
\hline Age, years & $65 \pm 10$ & $67 \pm 11$ & $71 \pm 11$ \\
\hline $\operatorname{Men}(\%)$ & $184(72)$ & $46(57)$ & $95(65)$ \\
\hline BMI, $\mathrm{kg} / \mathrm{m}^{2}$ & $28.4 \pm 4.6$ & $30.2 \pm 4.7$ & $28.5 \pm 6.0$ \\
\hline Total cholesterol, mg/dL & $193 \pm 48$ & $201 \pm 61$ & $174 \pm 48$ \\
\hline LDL cholesterol, mg/dL & $109 \pm 40$ & $111 \pm 46$ & $95 \pm 35$ \\
\hline HDL cholesterol, mg/dL & $49 \pm 15$ & $49 \pm 17$ & $47 \pm 14$ \\
\hline Triglycerides, mg/dL & $144(104-203)$ & $165(110-240)$ & $130(90-187)$ \\
\hline Systolic blood pressure, $\mathrm{mm} \mathrm{Hg}$ & $136.1 \pm 19.1$ & $135.2 \pm 16.1$ & $137.3 \pm 22.8$ \\
\hline Diastolic blood pressure, $\mathrm{mm} \mathrm{Hg}$ & $71.7 \pm 9.8$ & $70.1 \pm 9.1$ & $68.7 \pm 10.7$ \\
\hline Pulse pressure, $\mathrm{mm} \mathrm{Hg}$ & $64.5 \pm 17.0$ & $65.4 \pm 15.6$ & $67.0 \pm 18.3$ \\
\hline Serum creatinine, $\mathrm{mg} / \mathrm{dL}$ & $1.1 \pm 0.5$ & $1.1 \pm 0.4$ & $1.4 \pm 1.2$ \\
\hline $\mathrm{eGFR}, \mathrm{mL} / \mathrm{min}$ & $66.5 \pm 18.9$ & $65.7 \pm 20.9$ & $62.0 \pm 24.1$ \\
\hline Ever smoker $(\%)$ & $217(85)$ & $68(85)$ & 109 (74) \\
\hline Hypertension (\%) & $177(70)$ & $57(71)$ & $113(77)$ \\
\hline Diabetes (\%) & $59(23)$ & $24(30)$ & $65(44)$ \\
\hline Dyslipidemia (\%) & $218(86)$ & $72(90)$ & $117(80)$ \\
\hline Statin use $(\%)$ & $128(50)$ & $44(55)$ & $69(46)$ \\
\hline CHD (\%) & $140(55)$ & $39(49)$ & $83(56)$ \\
\hline $\operatorname{CVD}(\%)$ & $76(30)$ & $31(39)$ & $57(39)$ \\
\hline Resting ABI & $0.72 \pm 0.25$ & $0.65 \pm 0.22$ & $0.69 \pm 0.41$ \\
\hline Post-exercise ABI & $0.48 \pm 0.27$ & $0.43 \pm 0.25$ & $0.40 \pm 0.27^{e}$ \\
\hline NT-pro-BNP, pg/mL & $109(52-297)$ & $177(71-411)$ & $425(116-1214)$ \\
\hline Log NT-pro-BNP & $4.79 \pm 1.35$ & $5.16 \pm 1.12$ & $5.93 \pm 1.61$ \\
\hline Walking distance, yards & $232.6 \pm 56.7$ & $104.5 \pm 23.3$ & $50.7 \pm 6.6$ \\
\hline
\end{tabular}

Abbreviations: BMI, body mass index; LDL, low-density lipoprotein; HDL, high-density lipoprotein; eGFR, estimated glomerular filtration rate; CHD, coronary heart disease; CVD, cerebrovascular disease; ABI, ankle-brachial index; NT-pro-BNP, $N$-terminal pro-B-type natriuretic peptide.

${ }^{a}$ Continuous variables are presented as mean \pm standard deviation, or median (interquartile ranges); categorical variables are presented as numbers (percentages).

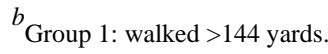

${ }^{c}$ Group 2: walked 60-144 yard.

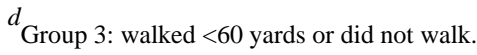

${ }^{e}$ A total of 18 patients with peripheral arterial disease underwent exercise testing in this group. 
Table 2

Association between Serum NT-Pro-BNP Levels and Walking Distance in Patients with Peripheral Arterial Disease by Ordinal Logistic Regression Analysis

\begin{tabular}{lcc}
\hline & Odds Ratio (95\% Confidence Interval) & $\boldsymbol{P}$ \\
\hline Unadjusted & $1.63(1.43-1.86)$ & $<.001$ \\
Model $1^{a}$ & $1.53(1.33-1.76)$ & $<.001$ \\
Model $2^{b}$ & $1.52(1.30-1.79)$ & $<.001$ \\
Model $3^{c}$ & $1.51(1.28-1.77)$ & $<.001$ \\
\hline
\end{tabular}

Abbreviations: NT-pro-BNP, $N$-terminal pro-B-type natriuretic peptide; eGFR, estimated glomerular filtration rate.

${ }^{a}$ Model 1: adjusted for age, sex, and body mass index (BMI).

${ }^{b}$ Model 2: adjusted for age, sex, BMI, smoking status, presence of diabetes, hypertension, dyslipidemia, coronary heart disease/cerebrovascular disease, eGFR, statin use, and pulse pressure.

${ }^{c}$ Model 3: adjusted for variables in model 2 plus resting ankle-brachial index. 
Table 3

Association between Serum NT-Pro-BNP Levels and Walking Distance in Patients with Peripheral Arterial Disease by Multiple Linear Regression Analysis ${ }^{a}$

\begin{tabular}{lcc}
\hline & $\beta^{b} \pm$ Standard Error & $\boldsymbol{P}$ \\
\hline Unadjusted & $-19.0 \pm 5.2$ & $<.001$ \\
Model $^{c}$ & $-11.9 \pm 5.4$ & .038 \\
Model $2^{d}$ & $-13.9 \pm 5.9$ & .020 \\
Model $^{e}$ & $-11.6 \pm 5.9$ & .049 \\
\hline
\end{tabular}

Abbreviations: NT-pro-BNP, $N$-terminal pro-B-type natriuretic peptide; eGFR, estimated glomerular filtration rate.

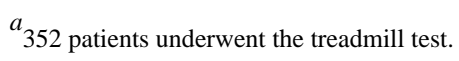

${ }^{b}$ The $\beta$ coefficients represent the predicted response (walking capacity in yards) for a 1 unit change in log NT-pro-BNP level.

${ }^{c}$ Model 1: adjusted for age, sex, and body mass index (BMI).

${ }^{d}$ Model 2: adjusted for age, sex, BMI, smoking status, presence of diabetes, hypertension, dyslipidemia, coronary heart disease/cerebrovascular disease, eGFR, statin use, and pulse pressure.

${ }^{e}$ Model 3: adjusted for variables in model 2 plus resting ankle-brachial index. 